\title{
A Game Variant of the Stopping Problem on Jump Processes with a Monotone Rule
}

\author{
Jun-ichi Nakagami \\ Department of Mathematics and Informatics, Faculty of Science, \\ Chiba University, Chiba 263-8522, Japan. \\ (nakagami@math.s.chiba-u.ac.jp) \\ Masami Kurano \\ Department of Mathematics, Faculty of Education, \\ Chiba University, Chiba 263-8522, Japan. \\ (kurano@math.e.chiba-u.ac.jp) \\ Masami Yasuda \\ Department of Mathematics and Informatics, Faculty of Science, \\ Chiba University, Chiba 263-8522, Japan. \\ (yasuda@math.s.chiba-u.ac.jp)
}

\begin{abstract}
A continuous-time version of the multivariate stopping problem is considered. Associated with vector valued jump stochastic processes, stopping problems with a monotone logical rule are defined under the notion of Nash equilibrium point. The existence of an equilibrium strategy and its characterization by integral equations are obtained. Illustrative examples are provided.
\end{abstract}

\section{Introduction}

In social life or in business, group decision making is often alleviated by cooperating with each individual's opinion in the whole group. How can we impose their opinion to the group decision? As one abstraction of such a situation, we shall try to propose a multivalued stopping game by introducing a monotone logical function to sum up each individual's opinion. The discrete-time case has already been discussed [4], [10]. Here we consider the continuous-time case, which is formulated as a multiobjective extension of Karlin's model [3] and a rule's extension of Sakaguchi's model [7]. As a related result, Presman and Sonin [6] have obtained the multiperson best choice problem on the Poisson stream but their rule of a decision to stop is different from ours. Szajowski and Yasuda [8] treat the case when the process is a Markov Chain.

The situation of our problem is as follows. A group of $p$ players observes a $p$ dimensional stochastic process. Each player can decide to stop or to continue the process 
at any time when the $p$-dimensional successive offers will have happened, and the individual declarations are summed to make the group decision for the process by using a monotone logical rule. When the process is stopped by the group of $p$ players, components of the stochastic process are given to each player as a reward, so that he wishes to make his expected gain as large as possible.

First, we introduce some definitions and notations to formulate our stopping problem in Section 2. Then, by preparing several lemmas, we show the existence of an equilibrium stopping strategy and obtain its characterization by an integral equation in Section 3. In Section 4, examples of the underlying model are given.

\section{Formulation}

We consider a $p$-dimensional vector valued stochastic process $\left\{X_{t} ; t \geq 0\right\}$ with $i$ th component $X_{t}^{i}$, adapted to $\mathcal{F}_{t}$ on a probability space $(\Omega, \mathcal{F}, P)$ where $\mathcal{F}_{t}$ is the $\sigma$-algebra generated by $\left\{X_{s} ; 0 \leq s \leq t\right\}$. Let us assume that the process $\left\{X_{t} ; t \geq 0\right\}$ is an independent jump process (see, Feller [2]), that is, there are two independent stochastic sequences $Z_{n}=\left(Z_{n}^{1}, Z_{n}^{2}, \ldots, Z_{n}^{p}\right)$ and $\tau_{n}, n=0,1,2, \ldots$, which satisfy $X_{t}=Z_{n}$ if $\tau_{n} \leq t<\tau_{n+1}$ for any $t, t \geq 0$, under the following assumption:

\section{Assumption 2.1.}

(a) $p$-Dimensional random vectors $Z_{n}=\left(Z_{n}^{1}, Z_{n}^{2}, \ldots, Z_{n}^{p}\right), n=0,1,2, \ldots$, are i.i.d. with a common distribution $F$ on $R^{p}$, where $R=(-\infty, \infty)$.

(b) $\tau_{0}=0$ a.s. and $\tau_{n}-\tau_{n-1}, n=1,2, \ldots$ are i.i.d. with a common distribution $G$ on $R_{+}$, where $R_{+}=[0, \infty)$ and $G(0)=0$.

(c) $\int_{R^{p}}|z| F(d z)<\infty$ and $\mu_{G}=\int_{R_{+}} t G(d t)<\infty$, where $|\cdot|$ is a norm on $R^{p}$.

In order to denote the declaration for each player $i(i=1, \ldots, p)$, when the process is $\left\{X_{t} ; t \geq 0\right\}$, let $\sigma^{i}(t, x)$ be a $\{0,1\}$-valued Borel measurable function on $R_{+} \times R^{p}$ with $\sigma^{i}(0, x)=1$. We call $\sigma^{i}=\sigma^{i}(\cdot, \cdot)$ an individual strategy for player $i$, and $\sigma=\left(\sigma^{1}, \ldots, \sigma^{p}\right)$ a strategy. The individual strategy $\sigma^{i}(t, x)$ may be interpreted as follows; when the amount $x$ of the offer has happened and the time interval remaining until termination is $t, \sigma^{i}(t, x)=$ 1(0) means player $i$ declares to stop (continue). In particular, $\sigma^{i}(0, x)=1$ means that any player $i$ must declare to stop when the time remaining until termination is 0 .

The individual declarations are summed up by a logical rule. A logical rule is a map $\pi:\{0,1\}^{p} \rightarrow\{0,1\}$ and is called monotone if $\pi(1, \ldots, 1)=1$ and $\pi\left(\sigma^{1}, \ldots, \sigma^{p}\right) \leq$ $\pi\left(\tilde{\sigma}^{1}, \ldots, \tilde{\sigma}^{p}\right)$ for $\sigma^{i} \leq \tilde{\sigma}^{i}(1 \leq i \leq p)$. A monotone logical rule includes a wide variety in a choice system such as a unanimity rule, an equal/unequal majority rule, and a hierarchical rule, some of which are given in the last section. For example, if no less than $r(\leq p)$ members in a group of $p$ players declare to stop, the group decision is to stop the process (equal majority rule). That is, $\pi\left(\sigma^{1}, \ldots, \sigma^{p}\right)=1(0)$ if $\sum_{i=1}^{p} \sigma^{i} \geq(<) r$. Refer also to our previous papers [4] and [10].

For a strategy $\sigma$, a monotone logical rule $\pi$ and a planning horizon $T$, a stopping time $t(T, \sigma, \pi)$ for the group of $p$ players is defined by

$$
t(T, \sigma, \pi)=\min \{\tau(\sigma, \pi), T\}
$$


where $\tau(\sigma, \pi)=$ the first $\tau_{k}$ such that $\pi\left(\sigma\left(T-\tau_{k}, X_{\tau_{k}}\right)\right)=1$ for $k \geq 0$. Note that $t(T, \sigma, \pi)$ means the first time that the declaration $\sigma^{i}$ of each player $i$ is summed up for the group of $p$ players, to stop the process by the rule $\pi$. Since the monotone logical rule $\pi$ is fixed, $\pi$ is suppressed in $t(T, \sigma, \pi)$ hereafter.

An expected reward of player $i$ for a strategy $\sigma$ is defined by

$$
u^{i}(T, \sigma)=E\left[X_{t(T, \sigma)}^{i}\right], \quad T \geq 0 .
$$

Since the problem is fundamentally formulated as a noncooperative game, a notion of Nash equilibrium point (see Nash [5] and Vorob'ev [9] ) can be utilized. A strategy ${ }^{*} \sigma=$ $\left({ }^{*} \sigma^{1}, \ldots,{ }^{*} \sigma^{p}\right)$ is equilibrium if, for each $i$,

$$
u^{i}\left(T,{ }^{*} \sigma\right) \geq u^{i}\left(T,{ }^{*} \sigma^{-i} \|_{\sigma^{i}}\right)
$$

for any individual strategy $\sigma^{i}$ and any $T \geq 0$, where

$$
{ }^{*} \sigma^{-i} \|_{\sigma^{i}}=\left({ }^{*} \sigma^{1}, \ldots,{ }^{*} \sigma^{i-1}, \sigma^{i},{ }^{*} \sigma^{i+1}, \ldots,{ }^{*} \sigma^{p}\right) .
$$

In this chapter, we will find an equilibrium strategy ${ }^{*} \sigma$ and the corresponding stopping time $t\left(T,{ }^{*} \sigma, \pi\right)$ given a monotone rule $\pi$.

\section{Lemmas and Theorems}

In this section, the existence of an equilibrium strategy and its characterization are obtained. First, we will derive the integral equation of $u(T, \sigma)=\left(u^{1}(T, \sigma), \ldots, u^{p}(T, \sigma)\right), T \geq$ 0 for given a strategy $\sigma$. Let $\mathcal{G}_{n}$ be the $\sigma$-algebra generated by $\left(Z_{k}, \tau_{k}\right), k=0,1, \ldots, n-$ 1 and $\tau_{n}$ for each $n$.

\section{Lemma 3.1.}

$$
E\left[X_{t(T, \sigma)}^{i} I_{\left\{t(T, \sigma) \geq \tau_{n}\right\}} \mid \mathcal{G}_{n}\right]=u^{i}\left(T-\tau_{n}, \sigma\right) I_{\left\{t(T, \sigma) \geq \tau_{n}\right\}} \quad \text { a.e. }
$$

where $I_{A}$ is the indicator for a set $A$.

Proof. By Assumption 2.1, it holds that

$$
t(T, \sigma)=t\left(T-\tau_{n}, \sigma\right)+\tau_{n} \quad \text { on } \quad\left\{t(T, \sigma) \geq \tau_{n}\right\}
$$

for some $n$ and that

$$
E\left[X_{t\left(T-\tau_{n}, \sigma\right)+\tau_{n}}^{i} \mid \mathcal{G}_{n}\right]=u^{i}\left(T-\tau_{n}, \sigma\right) \quad \text { a.e. }
$$

So, the proof is completed by noting $\left\{t(T, \sigma) \geq \tau_{n}\right\} \in \mathcal{G}_{n}$.

Let $Z=\left(Z^{1}, \ldots, Z^{p}\right)$ be a $p$-dimensional random variable whose distribution is $F$ and let $\mathcal{F}$ be the $\sigma$-algebra generated by $Z$. For any set $A \in \mathcal{F}$ and any $\alpha \in R$, define the operators $L^{i}, i=1, \ldots, p$, by

$$
L^{i}(A ; \alpha)=E\left[Z^{i} I_{A}\right]+\alpha P\left(A^{c}\right) .
$$


Lemma 3.2. For each $i(i=1, \ldots, p), u^{i}(T)=u^{i}(T, \sigma)$ satisfies the following integral equation:

$$
u^{i}(T)=L^{i}\left(\left\{\pi\left(\sigma_{(T, Z)}\right)=1\right\} ; G \circ u^{i}(T)\right)
$$

where $\sigma_{(T, Z)}=\left(\sigma_{(T, Z)}^{1}, \ldots, \sigma_{(T, Z)}^{p}\right)$ and $G \circ u^{i}(T)=\int_{0}^{T} u^{i}(T-s) G(d s)$.

Proof. By Assumption 2.1, we have

$$
u^{i}(T)=E\left[Z_{0}^{i} I_{\{t(T, \sigma)=0\}}\right]+E\left[X_{t(T, \sigma)}^{i} I_{\left\{t(T, \sigma) \geq \tau_{1}\right\}}\right] .
$$

From Lemma 3.1, it holds that

$$
E\left[X_{t(T, \sigma)}^{i} I_{\left\{t(T, \sigma) \geq \tau_{1}\right\}} \mid \mathcal{G}_{1}\right]=u^{i}\left(T-\tau_{1}\right) I_{\left\{t(T, \sigma) \geq \tau_{1}\right\}} \quad \text { a.e. }
$$

Thus, noting $\{t(T, \sigma)=0\}=\left\{\pi\left(\sigma_{\left(T, Z_{0}\right)}\right)=1\right\}$ and $\left\{t(T, \sigma) \geq \tau_{1}\right\}=\left\{\pi\left(\sigma_{\left(T, Z_{0}\right)}\right)=1\right\}^{c}$, (13.3) follows from (13.4), replacing $Z_{0}$ by $Z$.

To show the existence of an equilibrium strategy, we need several further lemmas.

Let $S$ be the set of all $\{0,1\}$-valued Borel measurable functions on $R_{+} \times R^{p}$. For any number $\alpha \in R$ and $i(i=1, \ldots, p)$, define $\sigma^{i}[\alpha] \in S$ by $\sigma^{i}[\alpha]=1$ if $Z^{i} \geq \alpha,=0$ otherwise, which is called an individual strategy of a control-limit-type.

For any $\left(\sigma^{1}, \ldots, \sigma^{p}\right) \in S^{p}$, let us denote $\pi(\sigma)=\pi\left(\sigma_{(T, Z)}^{1}, \ldots, \sigma_{(T, Z)}^{p}\right)$ for simplicity.

Lemma 3.3. For any $\alpha \in R$ and $\left(\sigma^{1}, \ldots, \sigma^{p}\right) \in S^{p}$,

$$
L^{i}(\{\pi(\sigma)=1\} ; \alpha) \leq L^{i}\left(\left\{\pi\left(\sigma^{-i} \|_{\sigma^{i}[\alpha]}\right)=1\right\} ; \alpha\right) .
$$

Proof. Since $\pi$ is monotone, we have $\pi\left(\sigma^{1}, \ldots, \sigma^{i-1}, 1, \sigma^{i+1}, \ldots, \sigma^{p}\right) \geq \pi\left(\sigma^{1}, \ldots, \sigma^{p}\right) \geq$ $\pi\left(\sigma^{1}, \ldots, \sigma^{i-1}, 0, \sigma^{i+1}, \ldots, \sigma^{p}\right)$ for all $\sigma \in S^{p}$. Thus, from the definition of $\sigma^{-i} \|_{\sigma^{i}[\alpha]}$, it follows that

$$
\left\{Z^{i}-\alpha \geq 0, \pi\left(\sigma^{-i} \|_{\sigma^{i}[\alpha]}\right)=1\right\} \supset\left\{Z^{i}-\alpha \geq 0, \pi(\sigma)=1\right\}
$$

and

$$
\left\{Z^{i}-\alpha<0, \pi\left(\sigma^{-i} \|_{\sigma^{i}[\alpha]}\right)=1\right\} \subset\left\{Z^{i}-\alpha<0, \pi(\sigma)=1\right\},
$$

which implies

$$
E\left[\left(Z^{i}-\alpha\right) I_{\{\pi(\alpha)=1\}}\right] \leq E\left[\left(Z^{i}-\alpha\right) I_{\left\{\pi\left(\sigma^{-i} \|_{\sigma^{i}[\alpha]}\right)=1\right\}}\right] .
$$

So, the proof is completed by noting $L^{i}(A ; \alpha)=E\left(\left[Z^{i}-\alpha\right) I_{A}\right]+\alpha$ for all $A \in \mathcal{F}$.

Lemma 3.4. For any $\alpha, \beta \in R$, with $\alpha \geq \beta$ and $\left(\sigma^{1}, \ldots, \sigma^{p}\right) \in S^{p}$,

$$
L^{i}\left(\left\{\pi\left(\sigma^{-i} \|_{\sigma^{i}[\alpha]}\right)=1\right\} ; \alpha\right) \geq L^{i}\left(\left\{\pi\left(\sigma^{-i} \|_{\sigma^{i}[\beta]}\right)=1\right\} ; \beta\right) .
$$

Proof. By Lemma 3.3, it holds that

$$
L^{i}\left(\left\{\pi\left(\sigma^{-i} \|_{\sigma^{i}[\alpha]}\right)=1\right\} ; \alpha\right) \geq L^{i}\left(\left\{\pi\left(\sigma^{-i} \|_{\sigma^{i}[\beta]}\right)=1\right\} ; \alpha\right) .
$$

Since $\alpha \geq \beta$, then

$$
L^{i}\left(\left\{\pi\left(\sigma^{-i} \|_{\sigma^{i}[\beta]}\right)=1\right\} ; \alpha\right) \geq L^{i}\left(\left\{\pi\left(\sigma^{-i} \|_{\sigma^{i}[\beta]}\right)=1\right\} ; \beta\right),
$$


so that (13.5) follows from (13.6).

Lemma 3.5. For any fixed $i$ and any strategies $\sigma=\left(\sigma^{1}, \ldots, \sigma^{p}\right) \in S^{p}$, let us consider the following integral equation with respect to $v(T):=v^{i}(T)$, for simplicity :

$$
v(T)=L^{i}\left(\left\{\pi\left(\sigma^{-i} \|_{\sigma^{i}[G \circ v(T)]}\right)=1\right\} ; G \circ v(T)\right)
$$

for $T \geq 0$. Then, we have:

(i) The solution $v(T)$ exists uniquely in $L^{1}([0, \infty), d G)$; and

(ii) $v(T) \geq u^{i}(T ; \sigma)$ for $T \geq 0$.

Proof. First we shall show the uniqueness of the solution of (13.7). Let $\alpha=G \circ v(T)$ and $\alpha^{\prime}=G \circ v^{\prime}(T)$, where $v(T)$ and $v^{\prime}(T)$ are two solutions of $(13.7)$ in $L^{1}([0, \infty), d G)$. We generally assume $\alpha \geq \alpha^{\prime}$. Then, since

$$
\pi\left(\sigma^{-i} \|_{\sigma^{i}[\alpha]}\right)=\pi\left(\sigma^{-i} \|_{\sigma^{i}\left[\alpha^{\prime}\right]}\right) \quad \text { on } \quad\left\{Z^{i} \leq \alpha^{\prime}\right\} \cup\left\{Z^{i}>\alpha\right\},
$$

we have

$$
\begin{aligned}
& E\left[Z^{i} I_{\left\{\pi\left(\sigma^{-i} \| \sigma^{i}[\alpha]\right)=1\right\}}\right]-E\left[Z^{i} I_{\left\{\pi\left(\sigma^{-i} \| \sigma^{i}\left[\alpha^{\prime}\right]\right)=1\right\}}\right] \\
= & E\left[Z^{i} I_{\left\{\alpha^{\prime}<Z^{i} \leq \alpha, \pi\left(\sigma^{-i} \| \sigma^{i}[\alpha]\right)=1\right\}}\right]-E\left[Z^{i} I_{\left\{\alpha^{\prime}<Z^{i} \leq \alpha, \pi\left(\sigma^{-i} \| \sigma^{i}\left[\alpha^{\prime}\right]\right)=1\right\}}\right] \\
\leq & \alpha P\left\{\alpha^{\prime}<Z^{i} \leq \alpha, \pi\left(\sigma^{-i} \|_{\sigma^{i}[\alpha]}\right)=1\right\}-\alpha^{\prime} P\left\{\alpha^{\prime}<Z^{i} \leq \alpha, \pi\left(\sigma^{-i} \|_{\sigma^{i}\left[\alpha^{\prime}\right]}\right)=1\right\} \\
\leq & \alpha P\left\{\pi\left(\sigma^{-i} \|_{\sigma^{i}[\alpha]}\right)=1\right\}-\alpha^{\prime} P\left\{\pi\left(\sigma^{-i} \|_{\sigma^{i}\left[\alpha^{\prime}\right]}\right)=1\right\} .
\end{aligned}
$$

It follows from (13.7) that $v(T)-v^{\prime}(T) \leq \alpha-\alpha^{\prime}$. Thus, we have $0 \leq v(T)-v^{\prime}(T) \leq G \circ$ $v(T)-G \circ v^{\prime}(T)$ from Lemma 4.4, which implies

$$
\left|v(T)-v^{\prime}(T)\right| \leq \int_{0}^{T}\left|v(T-s)-v^{\prime}(T-s)\right| G(d s) \quad \text { for all } \quad T \geq 0 .
$$

By the well-known Gronwall-Bellman's theorem (see, e.g. Bellman [1]), we obtain the result

$$
v(T)=v^{\prime}(T) \quad \text { for all } \quad T \geq 0
$$

in $L^{1}([0, \infty), d G)$.

Next, we shall show the existence of the solution of (13.7). For any strategy $\sigma$ it holds from Lemma 3.2 that

$$
u^{i}(T ; \sigma)=L^{i}\left(\left\{\pi\left(\sigma_{(T, Z)}\right)=1\right\} ; G \circ u^{i}(T ; \sigma)\right) .
$$

Now putting $\alpha_{1}^{i}=G \circ u^{i}(T, \sigma)$ and $\sigma_{1}^{i}=\sigma^{i}\left[\alpha_{1}^{i}\right]$, we define

$$
u_{1}^{i}(T)=L^{i}\left(\left\{\pi\left(\sigma^{-i} \|_{\sigma_{1}^{i}}\right)=1\right\} ; \alpha_{1}^{i}\right) .
$$

Then we observe from Lemma 3.3 that

$$
u_{1}^{i}(T) \geq u_{1}^{i}(T ; \sigma) .
$$

If we define, recursively, for each $n \geq 2$,

$$
u_{n}^{i}(T)=L^{i}\left(\left\{\pi\left(\sigma^{-i} \|_{\sigma_{n}^{i}}\right)=1\right\} ; \alpha_{n}^{i}\right),
$$




$$
\alpha_{n}^{i}=G \circ u_{n-1}^{i}(T) \quad \text { and } \quad \sigma_{n}^{i}=\sigma^{i}\left[\alpha_{n}^{i}\right]
$$

we see that

$$
u_{n}^{i}(T) \geq u_{n-1}^{i}(T)
$$

by applying Lemma 3.4. Hence by the monotone convergence theorem, when $n \rightarrow \infty$ in (13.10), it holds that the limit $u^{i}(T):=\lim _{n \rightarrow \infty} u_{n}^{i}(T)$ equals a solution $v(T):=v^{i}(T)$ of (13.7) in $L^{1}([0, \infty), d G)$. Clearly, (ii) holds from (13.9) and (13.11).

Condition 3.1. There are $v^{i}(T) \in L^{1}([0, \infty), d G), i=1, \ldots, p$, which satisfy the following $p$ simultaneous integral equations:

$$
v^{i}(T)=L^{i}\left(\left\{\pi\left(^{*} \sigma\right)=1 ; G \circ v^{i}(T)\right), \quad i=1, \ldots, p, \quad T \geq 0,\right.
$$

where ${ }^{*} \sigma=\left({ }^{*} \sigma^{1}, \ldots,{ }^{*} \sigma^{p}\right)$ and ${ }^{*} \sigma^{i}={ }^{*} \sigma_{(T, Z)}^{i}=\sigma^{i}\left[G \circ v^{i}(T)\right]$.

We are now ready to prove the main theorem.

Theorem 3.1. Under Condition 1, it holds that:

(i) $u^{i}\left(T,{ }^{*} \sigma\right)=v^{i}(T), \quad i=1, \ldots, p$ for $T \geq 0$.

(ii) ${ }^{*} \sigma$ is an equilibrium strategy.

Proof . (i) By Lemma 3.2, $u^{i}\left(T ;{ }^{*} \sigma\right)$ satisfies (13.12). Thus, from (i) of Lemma 3.5, the uniqueness of the solution of (13.12) implies (i) of Theorem 3.1. Also, (ii) follows from (ii) of Lemma 3.5.

Remark 3.1. Theorem 3.1 says that under Condition 3.1 there exists an equilibrium strategy of control-limit-type, whose threshold for each player $i$ is $\alpha^{i}=G \circ v^{i}(t)$ when the remaining time interval until termination is $t$.

Remark 3.2. In most cases the direct verification of Condition 3.1 seems to be difficult. However, if $G(T)<1$ for all $T>0$ as the case that $G(d s)=\lambda e^{-\lambda s} d s, \lambda>0$ (an exponential distribution), (13.12) has a unique solution $v(T)=\left(v^{1}(T), \ldots, v^{p}(T)\right)$ in $L^{\infty}[0, \infty)^{p}$, where $L^{\infty}[0, \infty)$ denotes the set of all bounded Borel measurable functions on $[0, \infty)$. This result is used as the example in the next section. In fact, we define the map $U: L^{\infty}[0, \infty)^{p} \rightarrow L^{\infty}[0, \infty)^{p}$ by

$$
U u(T)=\left(L^{1}\left(\pi\left\{\sigma_{u(T)}\right)=1\right\} ; G \circ u^{1}(T), \ldots L^{p}\left(\pi\left\{\sigma_{u(T)}\right)=1\right\} ; G \circ u^{p}(T)\right),
$$

where $u(T)=\left(u^{1}(T), \ldots, u^{p}(T)\right) \in L^{\infty}[0, \infty)^{p}$ and

$$
\sigma_{u(T)}=\left(\sigma^{1}\left[G \circ u^{1}(T)\right], \ldots, \sigma^{p}\left[G \circ u^{p}(T)\right]\right) .
$$

Then, by the same way as in the proof of Lemma 3.5, we get

$$
\left\|U u-U u^{\prime}\right\|_{T} \leq G(T)\left\|u-u^{\prime}\right\|_{T} \quad \text { for any } u, u^{\prime} \in L^{\infty}[0, \infty)^{p} \text { and } T \geq 0,
$$

where $\|u\|_{T}=\max _{1 \leq i \leq p} \sup _{0 \leq t \leq T}\left|u^{i}(t)\right|$ for $u(T) \in L^{\infty}[0, \infty)^{p}$.

The above discussion shows that $U$ is a contraction w.r.t. the norm $\|\cdot\|_{T}$, so that $U$ has a unique fixed point $v_{T} \in L^{\infty}[0, \infty)^{p}$. Since $T$ is arbitrary, $v:=\lim _{T \rightarrow \infty} v_{T}$ satisfies (13.12). Also, the uniqueness of the solution of (13.12) follows from Lemma 3.5. 
Remark 3.3. If the observation cost is incurred at each arrival time of offers, a $p$ dimensional random vector (net profit) $Z_{n}=\left(Z_{n}^{1}, \ldots, Z_{n}^{p}\right)$ is defined by

$$
Z_{n}=Y_{n}-(n+1) c
$$

where $Y_{n}=\left(Y_{n}^{1}, \ldots, Y_{n}^{p}\right)$ are i.i.d. with a common distribution $F$ on $R^{p}$ and $c=$ $\left(c^{1}, \ldots, c^{p}\right)$ is a constant observation cost.

The corresponding $p$-simultaneous integral equations for (13.12) reduces, for $T \geq 0$,

$$
\left.v^{i}(T)+c^{i}=L^{i}\left(\left\{\pi{ }^{*} \sigma\right)=1\right\} ; G \circ v^{i}(T)\right), \quad i=1, \ldots, p .
$$

Then we can prove in identical fashion that for a solution $v^{i}(T) \in L^{1}([0, \infty), d G), i=$ $1, \ldots, p$, of (13.13) the same theorem as Theorem 3.1 holds.

Remark 3.4. When $G$ is a degenerate distribution with total mass at unity, the integral equation (13.13) becomes

$$
v^{i}(T)+c^{i}=L^{i}\left(\left\{\pi\left(^{*} \sigma\right)=1\right\} ; v^{i}(T-1)\right), \quad i=1, \ldots, p,
$$

where ${ }^{*} \sigma=\left({ }^{*} \sigma^{1}, \ldots,{ }^{*} \sigma^{p}\right)$ and ${ }^{*} \sigma_{(T, Z)}^{i}={ }^{*} \sigma^{i}\left[v^{i}(T-1)\right]$. Thus, if we define a sequence $\left\{v_{n}^{i}\right\}_{n=0,1, \ldots}$ for $i=1, \ldots, p$, by

$$
\begin{aligned}
v_{0}^{i} & =E\left[Z^{i}\right], \\
v_{n}^{i} & =L^{i}\left(\left\{\pi\left({ }^{*} \sigma\right)=1\right\} ; v_{n-1}^{i}\right), n=1,2, \ldots,
\end{aligned}
$$

recursively, then we observe that

$$
v^{i}(T)=v_{n}^{i} \quad \text { if } \quad n \leq T<n+1 \quad \text { for some } n .
$$

Assuming that $c^{i}>0$ for all $i$, it follows that $v^{i}:=\lim _{T \rightarrow \infty} v^{i}(T)$ exists. Now, as $T \rightarrow \infty$ in (13.14), we obtain

$$
\left.E\left[\left(Z^{i}-v^{i}\right)^{+} P\left(\left\{\pi\left({ }^{*} \sigma^{-i} \|_{1}\right)=1\right\} \mid Z^{i}\right)\right]-E\left[\left(Z^{i}-v^{i}\right)^{-} P\left(\left\{\pi{ }^{*} \sigma^{-i} \|_{0}\right)=1\right\} \mid Z^{i}\right)\right]=c^{i},
$$

where ${ }^{*} \sigma^{-i} \|_{k}=\left({ }^{*} \sigma^{1}, \ldots,{ }^{*} \sigma^{i-1}, k,{ }^{*} \sigma^{i+1}, \ldots,{ }^{*} \sigma^{p}\right)$ for each $k=0,1$. We note that $(13.16)$ corresponds to $(3.1)$ of [10].

\section{Examples}

In this section, we provide some examples involving the two-person stopping problem $(p=$ 2 ) with the unanimity and simple majority rule as the typical ones of a monotone logical rule.

Example 4.1 (The Unanimity Rule). Let us consider a unanimity rule, that is, decide to stop only both of players' opinion is stop. Let define $\pi\left(\sigma^{1}, \sigma^{2}\right), \sigma^{1}, \sigma^{2} \in\{0,1\}$ by $\pi\left(\sigma^{1}, \sigma^{2}\right)=1$ if $\sigma^{1}=\sigma^{2}=1,=0$ otherwise. Then the integral equation (13.12) of $v^{i}(T), T \geq 0$ becomes

$$
v^{i}(T)=\bar{v}_{T}^{i}+\iint_{D}\left(z^{i}-\bar{v}_{T}^{i}\right) F\left(d z^{1}, d z^{2}\right), \quad i=1,2
$$


where $D=\left\{\left(z^{1}, z^{2}\right) ; z^{1} \geq \bar{v}_{T}^{1}\right.$ and $\left.z^{2} \geq \bar{v}_{T}^{2}\right\}$. The equilibrium strategy is of control-limittype and the threshold of player $i$ is $\bar{v}_{T}^{i}=G \circ v^{i}(T)=\int_{(0, T]} v^{i}(T-s) G(d s), i=1,2$.

If $Z^{1}$ and $Z^{2}$ are i.i.d. with a common distribution $F(z)$, then $v(T):=v^{1}(T)=v^{2}(T)$ for all $T \geq 0,(13.17)$ becomes

$$
v(T)=\bar{v}_{T}+\left(1-F\left(\bar{v}_{T}\right)\right) \int_{\bar{v}_{T}}^{\infty}(1-F(z)) d z,
$$

where $\bar{v}_{T}=\int_{(0, T]} v(T-s) G(d s)$.

Now suppose $G(d s)=\lambda e^{-\lambda s} d s, \lambda>0$, that is, the time interval between successive offers is exponentially distributed. Then since $d \bar{v}_{T} / d T=\lambda\left(v(T)-\bar{v}_{T}\right)$, we have the following differential equation from (13.18):

$$
\frac{d \bar{v}_{T}}{d T}=\lambda\left(1-F\left(\bar{v}_{T}\right)\right) \int_{\bar{v}_{T}}^{\infty}(1-F(z)) d z
$$

which corresponds to (10) of Sakaguchi [7].

Example 4.2 (The Simple Majority Rule). A simple majority rule $\pi\left(\sigma^{1}, \sigma^{2}\right), \sigma^{1}, \sigma^{2} \in$ $\{0,1\}$ for $p=2$ is defined by $\pi\left(\sigma^{1}, \sigma^{2}\right)=1$ if $\sigma^{1}+\sigma^{2} \geq 1,=0$ otherwise. If $Z^{1}$ and $Z^{2}$ are nonnegative and i.i.d. with a common distribution $F(z)$, then $v(T):=v^{1}(T)=v^{2}(T)$, and (3.13) becomes

$$
v(T)=\bar{v}_{T}+\iint_{D}\left(z^{1}-\bar{v}_{T}\right) F\left(d z^{1}\right) F\left(d z^{2}\right),
$$

where $D=\left\{\left(z^{1}, z^{2}\right) ; z^{1} \geq \bar{v}_{T}\right.$ or $\left.z^{2} \geq \bar{v}_{T}\right\}$. The equilibrium strategy is of control-limit-type and the threshold of player $i$ is

$$
\bar{v}_{T}=G \circ v(T)=\int_{(0, T]} v(T-s) G(d s) .
$$

Then, we have from Assumption 2.1 that $\mu_{F}=\int_{R} x F(d x)<\infty$,

$$
v(T)=\mu_{F}-F\left(\bar{v}_{T}\right) \int_{\left[0, \bar{v}_{T}\right]} z F(d z)+\bar{v}_{T}\left\{F\left(\bar{v}_{T}\right)\right\}^{2} .
$$

Since

$$
\int_{[0, y]} z F(d z)=-y[1-F(y)]+\int_{0}^{y}(1-F(z)) d z \quad \text { for all } y>0
$$

we obtain that

$$
v(T)=\mu_{F}+F\left(\bar{v}_{T}\right) \int_{0}^{\bar{v}_{T}} F(z) d z
$$

so that

$$
\bar{v}_{T}=\int_{(0, T]} G(d s)\left\{\mu_{F}+F\left(\bar{v}_{T-s}\right) \int_{0}^{\bar{v}_{T-s}} F(z) d z\right\} .
$$

Next, suppose $G(d s)=\lambda e^{-\lambda s} d s$. Then, by elementary calculus, (13.21) becomes

$$
\bar{v}_{T} \cdot \exp \{\lambda T\}=\lambda \int_{0}^{T} e^{\lambda s} d s\left\{\mu_{F}+F\left(\bar{v}_{s}\right) \int_{0}^{\bar{v}_{s}} F(z) d z\right\} .
$$


By taking the derivative of both sides of (13.22) with respect to $T$, we have the following differential equation:

$$
\frac{d \bar{v}_{T}}{d T}=\lambda\left\{\mu_{F}-\bar{v}_{T}+F\left(\bar{v}_{T}\right) \int_{0}^{\bar{v}_{T}} F(z) d z\right\} .
$$

Thus, by rewriting (13.23),

$$
d \bar{v} /\left\{\mu_{F}-\bar{v}+F(\bar{v}) \int_{0}^{\bar{v}} F(z) d z\right\}=\lambda d t
$$

from which we obtain the inverse function of $\bar{v}_{T}, T(\bar{v})$, given by

$$
T(\bar{v})=\lambda^{-1} \int_{0}^{\bar{v}} M^{-1}(\xi) d \xi
$$

where $M(\xi)=\mu_{F}-\xi+F(\xi) \int_{0}^{\xi} F(z) d z$.

For a numerical example, supposing $F(z)=z, 0 \leq z \leq 1$, from (13.24) we get

$$
T(\bar{v})=2 \lambda^{-1} \int_{0}^{\bar{v}} 1 /\left(\xi^{3}-2 \xi+1\right) d \xi,
$$

so that

$$
\lim _{T \rightarrow \infty} \bar{v}_{T}=(\sqrt{5}-1) / 2(\approx 0.6180) \quad \text { and } \quad \lim _{T \rightarrow \infty} v(T)=(\sqrt{5}-1) / 2,
$$

which are the threshold of the control-limit strategy and the expected reward for the infinite horizon problem (refer to Table 3.1 of [10] for the discrete time case) . 


\section{Acknowledgment.}

The authors wish to express their thanks to the referees for helpful comments and suggestions to improve this chapter.

\section{References}

[1] Bellman, R. Stability Theory of Differential Equations, McGraw-Hill, New York, 1953.

[2] Feller, W. An Introduction to Probability Theory and its Applications, II, Wiley, New York, 1966.

[3] Karlin, S. Stochastic Models and Optimal Policy for Selling an Asset, Chapter 9 in Studies in Applied Probability and Management Sciences, Stanford University Press, Stanford, CA, 1962.

[4] Kurano,M., Yasuda,M. and Nakagami,J. Multi-Variate Stopping Problem with a Majority Rule, Journal of the Operations Research Society of Japan, 23, 205-223(1980).

[5] Nash,J. Non-cooperative Game, Annals Mathematics, 54, 286-295(1951).

[6] Presman, E.L. and Sonin, I.M. Equilibrium Points in a Game Related to the Best Choice Problem, Theory of Probability and its Applications, 20, 770-781(1975).

[7] Sakaguchi,M. When to Stop: Randomly Appearing Bivariate Target Values, Journal of the Operational Research Society of Japan, 21, 45-57(1978).

[8] Szajowski,K. and Yasuda,M. Voting Procedure on Stopping Games of Markov Chain, In Stochastic Modeling in Innovative Manufacturing, (A.H.Christer, S.Osaki, and L.C.Thomas, eds.) Lecture Note in Economics and Mathematical System 445, Springer-Verlag, New York, 68-80(1997).

[9] Vorob'ev, N. N. Game Theory, Springer-Verlag, New York, 1977.

[10] Yasuda, M., Nakagami,J. and Kurano,M. Multivariate Stopping Problem with a Monotone Rule, Journal of the Operational Research Society of Japan, 25, 334$349(1982)$. 